\title{
Latest Developments in LED Standards
}

\begin{abstract}
The LED light sources can be stated as the most promising technology for the last decade from the lighting technology point of view. In order to compare LED chips and LED products from different manufacturers and achieve reproducible results, all of the manufacturers shall measure their LEDs or LED based products using same methodology. In this study, it is aimed to explain measurement and performance standards for LED chips and LED based Solid State Lighting products.
\end{abstract}

Keywords: LED, Solid State Lighting, Standards

\section{Introduction}

The LED light sources can be stated as the most promising technology for the last decade from the lighting technology point of view. Especially the usage of the highpower LEDs is increased rapidly with the developments in semi-conductor technology and electronics sector. The small sizes, high efficiency values, long lifetimes, the easy implementation to the automation systems and wide colour options are considered as the advantages of the LEDs over conventional light sources. On the other hand the small size of LEDs and high cooling needs make the mechanical and thermal design of the LED based luminaires more difficult. Without a good thermal design, the performance of the high-power LEDs can decrease significantly because of the rise in junction temperature. The junction temperature of the LEDs affects the luminous efficiency, luminous flux, efficacy and colour properties. Because of this temperature dependency, the performance of the LEDs can diverge significantly from the catalogue values especially for the high-power ones. Most of the catalogues do not contain the performance parameters for different temperatures and driving currents. The LED luminaire designs utilizing the catalogue values can lead to low-performance LED luminaires which decrease the reliability of LED based luminaires in the market. Therefore, the performance of a LED luminaire due to mechanical, thermal, optical and electrical design should be predicted beforehand.

One of the first steps of LED based luminaire design is the selection of the LED chips. In order to compare LED chips from different manufacturers, all of the manufacturers should measure their LEDs using same methodology. The methods should define standard conditions for the measurement of parameters like luminous flux, efficacy, colour properties, lifetime etc. Nevertheless, the different manufacturers' catalogues should give similar performance parameters and their changes for specific temperatures and driving currents. After LED chips assembled in a luminaire, the luminaire should be measured for optical, thermal and electrical characteristics. Likewise, in order to compare different luminaires, the measurement methods should be same for different types of luminaires. Since the performance of the LEDs are very tightly bonded with the mechanical design of the luminaire, new measurement methods should be defined. Also the measurement methods for conventional lamps and luminaires should be renewed for LED chips, LED lamps and LED based luminaires.

There exists some standards for the measurement of LED chips, LED lamps and LED luminaires optically, thermally or electrically. The first examples of these standards or guides are usually derived from electronic component standards or conventional measurement standards. Because of this situation, it can be said that the early standards are not clear and applicable enough. As an example, the early standards for the measurement of LED chips are not defined for different temperatures and different driving currents. The LED manufacturers usually specify the performance characteristics of LEDs for $25^{\circ} \mathrm{C}$ ambient temperature which makes the performance estimation for different temperatures difficult. Also because of the rapid development in LED technology, it had been tough to define a strict methodology. Especially when the LED lifetimes are of concern, the long measurement times reduces the practicability of LED lifetime measures. This situation brings along a great number of commercially available LED chips without proper lifetime measurement. With the latest standards, it is aimed to ensure shorter measurement times for predicting LED lifetimes.

In this study, it is aimed to explain current measurement standards for LED chips performances' and point out the differences from the conventional ones. Then the standards for LED modules and luminaires are explained. The current and new standards for the lifetime prediction of the LED chips and LED products are clarified.

\section{LED Chip Measurement Standards and Recommendations}

In this section the standards and recommendations for measuring performance and lifetime of LED chips are explained.

\section{CIE 127:2007 "Measurement of LEDs"}

One of the first document about the measurement of high power LEDs is the CIE's (CIE-Commission Internationale de L'eclairage) 127:2007 "Measurement of LEDs" technical report was published in 2007 [1]. The report is derived for high power LEDs from the document published in 1997 with the same name. It contains calculations and methods for photometric, radiometric and colorimetric measurement of single LED chips. It does not cover LED arrays, LED luminaires and organic LEDs (OLEDs). The LED chips connected to a DC source should be in thermal equilibrium with the environment. For this reason, the document does not cover the fast measurements during production stage. The ambient temperature is stated as $25^{\circ} \mathrm{C}$. A photometer or a spectrometer and/or a specktroradiometer can be used for measurements. An auxiliary light is needed in order to compensate the self-absorption of the LED and reflectance loss of the sphere coating. The measurement system should be calibrated with a reference source. The CIE 127:2007 does not cover measurement methods for different temperatures. Nevertheless it is important for detailed photometric, radiometric and spectroradiometric calculations and the details for explaining the measurement setups. It can be seen as a pioneering document. 
IES LM-85-14 "Electrical and Photometric Measurements of High-Power LEDs"

Another document for measuring high-power LEDs was published in 2014 by IES (IES-Illuminating Engineering Society) by the name of IES LM-85-14 "Electrical and Photometric Measurements of High-Power LEDs" [2]. It covers the measurement and characterization of LED chips, multi-chips and LEDs with remote phosphor for different temperatures. The methods are only valid for constant and pulsed DC currents. LED arrays, modules and AC LEDs are not in the scope of the document.

The required photometric and radiometric measurements for LM85 are; total luminous flux (Im), radiant optical power (W), total photon flux (mol/s) and efficacy $(\mathrm{Im} / \mathrm{W})$. The required colour property measurements are; colour coordinates $(x, y)$, correlated colour temperature (CCT) and colour rendering index (CRI). The required electrical measurements are; input $D C$ current and voltage, input power.

A climatic chamber or a thermoelectric plate should be used in order to control LED temperature. The sensitivity of the LEDs' properties to the temperature fluctuations makes the determination of the LED properties more difficult. For climatic chambers, 30 minutes of stabilization time is required. On the other hand 1 or 2 minutes of stabilization time is enough for the thermoelectric plates. If a pulsed DC source is to be used, the pulse time and the integration time of photometric/radiometric measurements should be selected that it will not increase the junction temperature of LED chip (i.e $5 \mathrm{~ms}$ ). In order to increase the accuracy of the pulsed measurements, the mean of multiple-measurement results can be used.

The different measurement setups and the measurement characteristics are explained below:

- Sphere-Spectroradiometer: total luminous flux, total radiant flux, photon flux, colour properties

- Sphere-Photometer: total luminous flux

- Gonio-Spectroradiometer: luminous intensity distribution, total luminous flux, total radiant flux, colour properties, chromaticity spatial uniformity

- Gonio-Photometer: luminous intensity distribution, total luminous flux

- Gonio-Colorimeter: luminous intensity distribution, total luminous flux, chromaticity spatial uniformity

LM85 is a guide developed for the measurement of high-power LEDs used for lighting products at different temperatures.

\section{IES LM-80-08 "Measuring Lumen Maintenance of LED Light Sources"}

LM80 describes the test methods to measure the lumen maintenance of LED chips, arrays and modules for a certain period of time. The document was published in 2008 by IES [3]. It does not cover LED luminaires or lamps. It only describes the procedures by which LED light sources can be operated under controlled conditions and produce comparable results. Any failure modes other than the maintenance of lumen output is not taken into consideration. This method does not allow to make estimation or extrapolation beyond the actual measurement hours.

The LED sources are tested with equal driving currents for three case temperatures; $55^{\circ} \mathrm{C}, 85^{\circ}$ and a third that manufacturer determines. The third value should be selected in a way that represents real life conditions. The photometric measurements of the LED sources should be conducted using same driving current. In order to check for colour variations during tests, it is recommended to use a spectroradiometer. The ambient temperature should be $25^{\circ} \mathrm{C} \pm 2^{\circ} \mathrm{C}$ during the photometric measurements and the LED sources should be cooled to room temperature beforehand. The sources should be used for at least 6.000 hours (10.000 hours are preferred) and measured with a sphere at a minimum of every 1000 hours. It is aimed to get lumen maintenance values for different driving currents and case temperatures.

Note: The required case temperature values reduced to two where at least one of the selected case temperature should be $55^{\circ} \mathrm{C}$ or $85^{\circ} \mathrm{C}$ by an addendum published by IES in $9^{\text {th }}$ January 2014.

\section{IES TM-21-11 "Life Estimation Test Method"}

The TM21 document was published by IES in 2011 [4]. It recommends a method to predict lumen maintenance of LED light sources using LM80 data. It defines setup, conditions and procedures for lumen maintenance testing of LED chips, arrays and modules. The lumen maintenance of a light source is defined as the time of hours which the light source maintains percentage of its initial light output. As an example, L70 is the time (in hours) when the light output has dropped to $70 \%$ of its initial light output. This time depends on the temperature, driving current, materials used etc. Therefore the lumen maintenance can vary from manufacturer to manufacturer and also between different LED light sources produced by same manufacturer.

For light sources measured for 6.000 hours up to 10.000 hours, the last 5.000 hours of LM80 data should be used. Data before 1.000 hours should not be used for curve fitting methods. For measurement durations greater than 10.000 hours, the last $50 \%$ of LM80 data should be used. For a sample size of 20 units or more, values must not be projected beyond 6 times the measurement duration. The lumen maintenance life projected should be given in format $L p$ (Dk) where $p$ is the percentage of initial light output and $\mathrm{D}$ is the total measurement hours of data. For example, for 6.000 hours of LM80 data Dk is equal to "6k". When the projected lumen maintenance data are greater than the 6 times the measurement duration, the results should be given with a greater sign. As an example with 6.000 hours data, if the projected life is greater than 36.000 hours the results should be given as; $L 70(6 k)>36.000$ hours. Also the case temperature and driving current should be given along with (at Ts $=55^{\circ} \mathrm{C}$, If $=350 \mathrm{ma}$ etc.). TM21 describes a method for predicting lifetime of LED light sources from LM80 data.

\section{LED Lamp and Luminaire Measurement Standards and Recommendations}

In this section the standards and recommendations for measuring performance and lifetime of LED lamps and luminaires are explained.

\section{ANSI NEMA C78.377 "Specifications for Chromaticity of Solid State Lighting Products"}

The document was published in 2008 and revised with small additions in 2011 by ANSI. "The purpose of this standard is to specify the range of chromaticities recommended for general lighting with solid state lighting (SSL) products, as well as to ensure that the white light chromaticities of the products can be communicated to consumers" [5]. It is only valid for LED based end products with integrated control systems, heating elements etc. Especially it is advised to be used for indoor LED lamps and LED based luminaires.

ANSI NEMA C78.377 describes nominal and target correlated colour temperatures (CCT). Target CCT value can be explained as the designed temperature value of the product. Nominal CCT value is the closest value to target 
CCT value (in $100 \mathrm{~K}$ intervals). Also the Duv parameter that defines the closest distance to the Planckian locus. The aim of this document is determination of the allowed intervals for the change in target CCT's due to deviations in the product line. The tolerance intervals are defined for both CCT and Duv values. ANSI NEMA suggests that the measurements should be in accordance with IES LM-79-08 "Electrical and Photometric Measurements of Solid-State Lighting Products" [6]

\section{IES LM-79-08 "Electrical and Photometric Measurements of Solid State Lighting Products"}

The LM79 was published in 2008 by IES and widely implemented by industry for photometric and electrical measurements of LED-based SSL (Solid State Lighting) products with control electronics and cooling elements incorporated [6]. A sphere-spectroradiometer/photometer or gonio-photometer may be used for the measurements. This document does not cover SSL products that require external elements to operate. The photometric measurements for LM80 are defined as; total luminous flux $(\mathrm{Im})$, luminous efficacy $(\mathrm{Im} / \mathrm{W})$, luminous intensity $(\mathrm{cd})$, chromaticity coordinates, correlated colour temperature (CCT) and colour rendering index (CRI). The electrical characteristics are defined as; input RMS AC voltage, input RMS AC current, input AC power, input voltage frequency and power factor (DC versions for DC powered products).

The performance of LEDs is directly related to the system that they are attached to due to thermal issues. For this reason, the photometric measurements are defined as "absolute" rather than "relative". The relative photometric measurements defined for luminaires utilizing a conventional light source are not applicable for LED based lighting systems since the thermal performance of the luminaire determines the performance of LED light source and LED light sources shouldn't be measured inside and outside the luminaire separately like conventional ones since the temperature of the LED chip would be different and may yield to erroneous results.

LM79 requires an ambient temperature of $25 \pm 1^{\circ} \mathrm{C}$ within $1 \mathrm{~m}$ radius of measurement setup. Also the air movement around the LED product should be minimized. The products should be mounted to the measurement device so that the heat conduction through the supporting objects causes negligible cooling effects. The orientation of the device under test should be the same with the orientation recommended by the manufacturer. The orientation of the product may change the temperature distribution inside the luminaire [7]. The products should reach stabilization and temperature equilibrium before the measurement. The variation (maximum-minimum) of at least 3 readings of the light output and electrical power over a period of 30 minutes, taken 15 minutes apart minutes apart should be less than $0.5 \%$.

The document is the most extensive document published for measurements of LED lamps and luminaires in a controlled environment.

\section{IES LM-84-14 "Measuring Luminous Flux and Colour Maintenance of LED Lamps, Light Engines and Luminaries"}

The LM84 was published by IES in 2014 [8]. The document extends the LM80 to LED lamps, light engines and luminaires. The document provides a method for measurement of light output and colour maintenance of above mentioned products. This method does not define estimations or extrapolation methods of maintenance data beyond the actual measurement time.
At system level, the components other than the LED light sources also contribute luminous flux decay and colour change over time. This method aims to achieve reproducible and comparable results between manufacturers. The ambient temperature during maintenance tests should be $25^{\circ} \mathrm{C} \pm 5^{\circ} \mathrm{C}$. The temperature measurement devices should be shielded from direct radiation. The relative humidity value should be less than $65 \%$ and the airflow around the product should be minimized. The device under test should be in the same orientation as it will be during its photometric measurement and should be same as the manufacturer's recommended orientation. The recommended voltage/current values should be used with a pure sinus AC power source. The products should be tested in accordance with LM79 with defined intervals [6]. The lifetime predictions using LM84 data are explained in the next section.

\section{IES TM-28-14 "Projecting Long-Term Luminous Flux} Maintenance of LED Lamps and Luminaires"

The TM28 was published by IES in 2014. The aim of the document is to provide guidance for sampling, test intervals and duration for projection of long term luminous flux maintenance of LED lamps and luminaires using LM84 data [9]. The method also utilizes LM80 data and TM21 calculations along with LM84 data. The TM28 offers two method, direct and combined extrapolation, namely. Direct extrapolation uses a minimum of 6.000 hours LM84 data measured. The intervals of measurements should not be more than 1.000 hours. Combined extrapolation method uses at least 3.000 hours of data collected initially 1.000 hours of and at least every 500 hours. Combined extrapolation utilizes the LED light source's LM80 data with TM21 calculations in accordance with LM84 measurements. The minimum number of sample products is three for direct extrapolation method and five for combined extrapolation method.

Direct extrapolation method is recommended where at least 6.000 hours of LM84 data are available. It uses a statistical curve-fitting method to extrapolate the luminous flux maintenance value to a certain level (i.e. $70 \%$ of initial light output). Depending on the sample size, the projected lifetime values can be a maximum of 6 times of actual LM84 measurement duration.

Combined extrapolation method utilizes minimum 6.000 hours of LM80 data and TM21 projection and a minimum of 3.000 hours LM84 data. Like the direct extrapolation method, depending on the sample size, the projected lifetime values can be a maximum of 6 times of actual LM84 measurement duration.

TM28 extends the TM21 to LED lamps and luminaires and provides a statistical approach to lifetime predictions of LED products.

\section{Discussion}

The number of LED based lighting systems is rapidly increasing. On the other hand, the adaptation of LED standards by manufacturers and laboratories is still deficient. This leads to problems in evaluating LED based products. Therefore, the LED based products are measured as conventional ones. The performance of LED based light sources are directly related to their temperatures, for this reason, they should be measured as a whole system, not separately. For this reason the photometric and radiometric measurements for LED products are defined as "absolute".

Due to rapid developments in LED technology, the measurement duration for LED lifetime predictions can be considered as long and may not be practical. Current versions of lifetime measurement methods may not able to 
cope with this rapid developments and R\&D processes. Shorter measurement durations are needed for lumen maintenance tests.

The most important parameter that determines LED light source and luminaire performance is the temperature. In order to obtain accurate results, thermally controlled measurement setups are needed. In order to compare LED chips between manufacturers and achieve reproducible results, the same methodology should be implemented by all manufacturers. The LED light sources' properties should be measured for different driving currents and temperatures to help designers and manufacturers to estimate the real world performance of a SSL product. Therefore the LED manufacturers' catalogues should contain LED performance data for different driving currents and temperatures. The thermal conditions of the ambient and measurement system should be tracked sensitively and stated in the measurement reports. The measurements without considering thermal equilibrium may yield to erroneous measurement results.

In this study the most "popular" documents for measuring LED light sources and LED based products are explained. It can be seen that, with the LED technology, the LED standards are rapidly developing, too. The conventional standards are still valid for the topics that the LED versions are missing. As a result, in order to evaluate the LED light sources and luminaires properly:

- LED light source catalogues should include the LED properties for different driving currents and junction temperatures.

- Validity of the measurement methods should be proved and the methods should provide comparable and reproducible results.
- The national standards and regulations should be prepared using acknowledged international documents

\section{REFERENCES}

[1] CIE Publication No: 127, Measurement of LEDs, (2007), Austria, ISBN 978-3-901-906-58-9

[2] IESNA IES LM-85-14, Approved Method for Electrical \& Photometric Measurements of High Power LEDs, (2014), New York, USA, ISBN 978-0-87995-289-1

[3] IESNA IES LM-80-08, Approved Method: Measuring Lumen Maintenance of LED Light Sources, (2008), New York, USA, ISBN 978-0-87995-227-3

[4] IESNA IES TM-21-11, Life Estimation Test Method, (2011), New York, USA, ISBN 978-0-87995-259-4

[5] ANSI ANSLG C78.377, Specifications for the Chromaticity of Solid State Lighting Products, (2011), USA

[6] IESNA IES LM-79-08, Approved Method: Electrical and Photometric Measurements of Solid-State Lighting Products, (2008), New York, USA, ISBN 978-0-87995-226-6

[7] Yurtseven M.B., Oğüş G., Onaygil S., The Effect of LED Luminaire Orientation on the Photometric Measurements Thermal Aspects, Balkan Light 2012, Belgrad, Serbia

[8] IESNA IES LM-84-14, Approved Method for Measuring Luminous Flux and Color Maintenance of LED Lamps, Light Engines, and Luminaires, (2014), New York, USA, ISBN 978-0-87995-288-4

[9] IESNA IES TM-28-14, Projecting Long-Term Luminous Flux Maintenance of LED Lamps and Luminaires, (2014), New York, USA, ISBN 978-0-87995-296-9

Authors: M. Berker Yurtseven, MSc, Prof. Dr. Sermin Onaygil, Istanbul Technical University, Energy Institute, Maslak, Istanbul, Turkey.byurtseven@itu.edu.tr 